\title{
Aprendizaje a través de resolución de problemas en el foro virtual con docentes en formación caso: ciencias naturales, área de educación ambiental
}

Sandra Lucía Guerra Gómez ${ }^{1}$

Ariel Adolfo Rodríguez Hernández ${ }^{2}$

\begin{abstract}
Resumen
La investigación buscó analizar y describir cómo se desarrolla el aprendizaje a través de resolución de problemas en un foro virtual, con los docentes en formación del programa de Licenciatura en Educación Básica de la UPTC. Para ello se realizó la investigación con estudiantes de séptimo semestre del programa en mención, pertenecientes a la sede de Tunja. La investigación aborda un análisis en torno al aprendizaje a través de la resolución de problemas en un ambiente virtual asincrónico como el foro. La información obtenida a través de la participación de los estudiantes se analizó con base en matrices de evaluación que determinaron los aspectos esperados en cuanto a la forma como se dio la resolución de problemas. Además, se utilizó una matriz de evaluación para la presentación de las ideas o aportes generados por los participantes, en la que se empleó la rúbrica Tigre.
\end{abstract}

Palabras clave: aprendizaje de las ciencias naturales, ambiente virtual de aprendizaje, resolución de problemas, foro virtual, problemas ambientales. 


\title{
Learning through problem solving in the virtual forum with teachers in training case: natural sciences, environmental education area
}

\begin{abstract}
This study sought to analyze and describe how learning takes place through resolution of problems in a virtual forum, with training and educational program in Elementary Education Bachelor of UPTC. The research was conducted with students from seventh semester of the program mentioned, belonging to the headquarters of Tunja. The investigation focuses on an analysis about learning through the resolution of problems in an asynchronous virtual environment like the forum. That information obtained through the participation of students was analyzed by using parent evaluation to determine expected aspects as to how to solve the problem. Also an evaluation matrix was used for the presentation of ideas or contributions from the participants in which we used the heading Tigre.
\end{abstract}

Key words: natural sciences learning, virtual learning environment, solving of problems, virtual forum, environmental problems.

Recibido: 16 de septiembre de 2010

Aceptado: 20 de octubre de 2010

\section{Introducción}

Esta investigación analiza el aprendizaje a través de la resolución de problemas en un foro virtual en los docentes en formación de séptimo semestre del programa de Licenciatura en Educación Básica de la Universidad Pedagógica y Tecnológica de Colombia. Con ello se busca, por una parte, aplicar la estrategia de resolución de problemas, y por otra, desarrollar esta estrategia en un foro virtual. A partir del análisis y la descripción alrededor de lo observado se intentó generar, en los docentes en formación, procesos de aprendizaje basados en la autogestión del conocimiento con interacción en ambientes virtuales.

El estudio resultó significativo en la medida en que toma en cuenta a los docentes en formación, quienes son los llamados a participar en los procesos educativos donde, de una u otra forma, intervienen actitudes y comportamientos que inciden 
de manera favorable o desfavorable en la formación de las nuevas generaciones. Para generar el aprendizaje de los estudiantes fue necesario crear espacios de discusión a través del diseño de un ambiente virtual asincrónico como el foro, donde pudieran encontrarse los elementos básicos de la participación y la manera como se maneja la información. A partir del análisis de su participación se pudo visualizar cómo se generan, interactúan y reproducen dichas intervenciones y la manera como se dan los procesos de aprendizaje en su autorregulación.

Abordar una problemática como la del aprendizaje constituye una interesante oportunidad de cuestionar lo que está ocurriendo en torno a la manera como se generan los conocimientos y las diversas intervenciones de los docentes en los diferentes escenarios educativos.

La descripción generada de la intervención en el desarrollo del foro virtual permite detectar los argumentos, el uso de la información, los procedimientos, la autonomía, la responsabilidad y el trabajo que se da al interior del proceso de aprendizaje de los y las docentes en formación. De una u otra manera, todo ello se estructura en un conjunto de prácticas y saberes que se convierten en procesos importantes como la formación de otros sujetos.

En este sentido se espera suscitar una reflexión frente a la problemática del aprendizaje, de modo que genere en los futuros docentes una conciencia y unos compromisos serios orientados a contrarrestar las concepciones que se tienen acerca de la transmisión del conocimiento. De esta forma es muy posible que a través del uso de las tecnologías de información y comunicación se den los espacios para que el aprendizaje se desarrolle de manera amena, abriendo espacios para la aprehensión de conocimientos. Así, el papel que cumplen las TIC adquiere toda su magnitud en razón de que su campo de acción se extiende más allá de lo puramente educativo. Para ello es primordial que quienes intervienen en los procesos de formación los introduzcan de manera efectiva al menos dentro del ámbito educativo.

\section{Marco de referencia}

Con la intención de suministrar un marco de referencia teórica que contextualice los postulados o teorías desde los que se pensó y dio curso a esta investigación, se establecieron unas bases mínimas estructuradas desde tres perspectivas de revisión. Se presentan en primera instancia las concepciones sobre aprendizaje y, particularmente, el de las ciencias naturales. En segunda instancia se aborda la estrategia de resolución de problemas desde el concepto de problema, y la enseñanza de la estrategia resolución de problemas. La tercera instancia es el foro virtual como ambiente de aprendizaje. En su conjunto, ellas tres dan el sentido que se intenta a la investigación. 
Un primer elemento imprescindible es el manejo del concepto de aprendizaje, necesario para la caracterización y descripción de los procesos de formación. Desde esta premisa el "aprendizaje" es considerado como un proceso que cumple un papel predominante en el ser humano, desde donde la persona aprende a manejar información, a construir conocimiento y a desarrollar competencias que le permitirán vivir con conciencia en el mundo y con una orientación hacia el fortalecimiento de su propio desarrollo.

Para describir el aprendizaje, particularmente de las ciencias naturales, se presentaron los diferentes modelos didácticos de la enseñanza de la ciencia, que permitieron visualizar de manera articulada los planteamientos relacionados con el papel del docente y de los estudiantes a lo largo del proceso de aprendizaje.

Un segundo elemento, y como estrategia de resolución de problemas en ciencias, consistió en tomar los referentes de Daniel Gil et al. (1989), quienes presentan un modelo que permite que los estudiantes desarrollen procesos adecuados cuando en él se enfrentan a problemas y/o ejercicios cerrados. En él se ven favorecidos aspectos relacionados con la metacognición y que mejoran la situación del aprendizaje escolar, ${ }^{3}$ mencionados a continuación:

- "Plantear situaciones problémicas" que a su vez "generen interés" teniendo en cuenta destrezas y actitudes de los estudiantes.

- "Estudio cualitativo de las situaciones problémicas" de tal manera que los estudiantes comiencen a estructurar un plan para la resolución del problema.

- "Orientar el tratamiento científico" hacia la formulación de hipótesis y elaboración de estrategias que las contrasten. Además de las estrategias propias para realizar el análisis de los resultados, se tienen en cuenta predicciones de las hipótesis generadas por el grupo de estudiantes o por la comunidad.

- "Plantear el manejo de nuevos conocimientos", tarea que requiere profundización de los conocimientos y consolidación de los mismos, en especial en las "relaciones ciencia, tecnología y sociedad", que de todas maneras dan la rigurosidad y el carácter que debe tener todo proceso científico.

- "Actividades de síntesis" con las que se plasman los resultados a través de la "elaboración de productos" que refuerzan el interés por el proceso y la "concepción de nuevos problemas" para favorecer la metacognición.

3 Gil y otros plantean que los estudiantes a través de esta estrategia pueden llegar a ser investigadores estructurados, es decir: "pueden llegar a abordar situaciones problemáticas de interés, interactuando con otros equipos y con el resto de la comunidad científica, representada por el profesor y los textos". 
Lo anterior, visto como estrategia para el aprendizaje, tiene una connotación especial de construcción o estructuración en los procesos de aprendizaje donde se potencializan los procesos mentales, la autorregulación y la autonomía de lo aprendido. Estas razones hacen que se considere importante el manejo de este modelo planteado por Gil y sus colaboradores 1989, para lo cual sus diferentes elementos suscitan mayor asimilación y cuestionamiento frente a los procesos de aprendizaje.

La tercera instancia: el foro virtual, es un referente clave pues a través de su utilización se pone en escena la estrategia de resolución de problemas, fundamental para explorar y comprender los procesos de aprendizaje que dinamizan los docentes en formación. Esto muestra, entre otras cosas, el papel tan importante que cumple un verdadero proceso de autoaprendizaje, en cuanto a que éste es el generador de la autonomía no sólo para potenciar las capacidades de cada persona sino también las diversas tareas, técnicas, medios, etc. que se necesitan para lograr un aprendizaje significativo.

La interactividad en un foro virtual debe ser muy clara. Además debe existir disposición de los participantes para la discusión, para la lectura de las intervenciones, para hacerse entender, para exponer y para argumentar puntos de vista. Galvis (2008) al respecto propone la utilización de la rúbrica Tigre, que es un acrónimo que recuerda fácilmente las cualidades al realizar un aporte en una discusión moderada, expresado en la tabla 1.

Tabla 1. Descripción de la rúbrica Tigre

T "Título diciente", que refleja el contenido del mensaje. Su carácter es llamativo y facilita la selección por parte de los participantes que deseen leerlo.

I "Ilación que es deseable entre aportes", como dice la Real Academia: "es la trabazón razonable y ordenada de las partes de un discurso." Se tienen presente aquellos elementos que sirven de base para construir ideas.

G Generación de discusión. Indica que la intervención debe ayudar a hacer más profunda la discusión, sembrando nuevas "semillas de discusión."

R "Redacción y buena presentación". Se debe tener en cuenta la elaboración de las ideas, pues estas pueden ser buenas pero no son captadas por problemas de puntuación, por encontrarse desordenadas, por colocar demasiadas abreviaturas o por ausencia de alguna palabra.

E Enriquecimiento de la discusión. Darle un valor agregado a la misma, pues no basta con decir "estoy de acuerdo con..." o repetir lo planteado por otros. 


\section{Metodología y enfoque de investigación}

Para alcanzar el objetivo de la investigación, se definieron las siguientes actividades:

- Identificar concepciones básicas relacionadas con el aprendizaje, la estrategia de resolución de problemas y el foro virtual como ambiente de aprendizaje.

- Determinar y diseñar los instrumentos de evaluación del aprendizaje a través de la resolución de problemas en un foro virtual con docentes en formación.

- Diseñar el curso virtual para realizar la prueba piloto de evaluación del aprendizaje a través de la resolución de problemas en un foro virtual.

- Desarrollar el curso de prueba para evaluar los resultados de la implementación del aprendizaje a través de la resolución de problemas en un foro virtual.

- Establecer las relaciones y resultados sobre la manera como se puede desarrollar el aprendizaje a través de la resolución de problemas en un foro virtual, para reflexionar acerca del aprovechamiento de los ambientes virtuales de aprendizaje y la proyección de nuevas estrategias de enseñanza en dichos ambientes.

\section{Fase de identificación de conceptualización básica}

En esta fase se indagaron e identificaron unas bases mínimas, estructuradas desde tres perspectivas de revisión. En primera instancia, las concepciones sobre aprendizaje. En segunda instancia, la estrategia de resolución de problemas, abordada desde el concepto de problema, resolución de problema y la enseñanza de la estrategia resolución de problemas. La tercera es el foro virtual como ambiente de aprendizaje. Esto incluye seleccionar las características de la estrategia de resolución de problemas y las de la utilización adecuada del foro virtual.

Fase de determinación y diseño de los instrumentos de evaluación del aprendizaje a través de la resolución de problemas en un foro virtual

Para la determinación y diseño de los instrumentos de evaluación del aprendizaje fue necesario tomar como elemento de abordaje la estrategia de resolución de problemas desarrollada por Daniel Gil (1989). La razón que hizo optar por esta estrategia fue permitir que los docentes en formación desarrollaran procesos 
de resolución más específicos y reales, generados en el contexto, favoreciendo aspectos relacionados con la creatividad y la metacognición.

Para desarrollar la estrategia de resolución de problemas se escogió el foro virtual, siguiendo a Mercer (2001), porque así se puede desarrollar el lenguaje académico o el discurso utilizado en la vida social e intelectual de una comunidad. Fundamentalmente, en el aprendizaje es importante el uso del lenguaje, los estilos convencionales de pensar con el lenguaje y las formas de presentación del conocimiento, tal como lo menciona Baracat (2006).

Para verificar las cualidades de los aportes en una discusión en el foro, se dispuso de la rúbrica Tigre, según Panqueva (2008), con la cual se favorece la calidad de los mensajes e intervenciones en el foro virtual. De esta manera el conocimiento y el lenguaje que se construyen y comparten en el foro se van convirtiendo en una alternativa privilegiada para el aprendizaje.

El diseño de los instrumentos (matrices de evaluación) se basó en los criterios establecidos por los autores Daniel Gil (para la estrategia resolución de problemas) y Álvaro Galvis (rúbrica Tigre), adaptando las particularidades de los mismos de tal manera que genere en los docentes en formación la autorregulación de sus intervenciones.

Fase de diseño del curso virtual para realizar la prueba piloto de evaluación del aprendizaje a través de la resolución de problemas en un foro virtual

Esta fase requirió solicitar a la oficina de educación virtual de la UPTC ${ }^{4}$ la creación del curso virtual denominado Aula interactiva de ciencias naturales, nombre que corresponde a una de las asignaturas del séptimo semestre del plan 834 del programa de Licenciatura en Educación Básica, cuyos contenidos programáticos desarrollan en la unidad 2 la temática correspondiente a resolución de problemas.

Luego de tener acceso al curso virtual y teniendo como base una introducción además de la identificación del curso, se estructuraron las unidades de estudio, resaltando para esta investigación la unidad 2, denominada "Proyecto de aula", cuyo indicador principal es "asumir la investigación en el aula y el contexto como un aspecto inherente a los procesos de desarrollo y construcción de conocimientos y competencias". A su vez se indicaron los materiales de estudio para el caso particular de la resolución del problema, se seleccionó un problema ambiental basado en el uso de plaguicidas, y como actividades para desarrollar la temática se implementó el foro. 
Se definió la población de estudio, esto es, el grupo $500^{5}$ correspondiente a la sede central de Tunja, que cursa la asignatura de Aula interactiva de ciencias naturales de la Licenciatura en Educación Básica de la Facultad de Estudios a Distancia de la UPTC.

De igual forma se definieron en esta fase las características para evidenciar el aprendizaje a través de la resolución de problemas en un foro virtual.

\section{Fase de desarrollo del curso}

Una vez que se han determinado y diseñado las matrices de evaluación para determinar el aprendizaje a través de la resolución de problemas en un foro virtual, se crea el curso virtual para implementar como actividad principal el foro y aplicar en éste la resolución de problemas. Igualmente se procedió a desarrollar el curso de prueba para analizar y describir el aprendizaje con base en los resultados obtenidos al incorporar esta estrategia.

\section{Fase de diagnóstico}

Esta fase final, como su nombre lo indica, buscó establecer el diagnóstico frente a la manera como se da el aprendizaje a través de la resolución de problemas en un foro virtual.

\section{Enfoque metodológico}

La actividad sistémica orientada a la comprensión de fenómenos educativos, a la toma de decisiones y al desarrollo organizado de conocimientos, hace que la modalidad de este trabajo de investigación sea cualitativa. Esto se basa en que la información obtenida con las intervenciones realizadas por el grupo de docentes en formación en el foro virtual donde se aplica la estrategia resolución de problemas da lugar a la comprensión del aprendizaje que se genera en ellos, al establecimiento de criterios de evaluación que determinan dicho proceso, que permite a su vez el análisis del fenómeno, entendiendo cómo, desde estas experiencias, se construye y se valida.

En este trabajo de investigación se asume el enfoque descriptivo, cuya finalidad es caracterizar un fenómeno (el aprendizaje), teniendo como referente unas variables (la estrategia resolución de problemas en un ambiente virtual asincrónico). Esta investigación no intenta, ni tiene la pretensión de explicar el porqué, sino de describir la manera como se da el aprendizaje a través de la resolución de problemas en el foro virtual, bajo la orientación de ciertos criterios evaluados que permiten el análisis y reflexión sobre dicho proceso en 
los docentes en formación. Por esta razón sólo se observa el objeto de estudio y se describe teniendo en cuenta características de acuerdo con el problema.

Se tendrá como base la información aportada por los participantes en las intervenciones realizadas en el foro virtual para explicar cómo se desarrolla el aprendizaje a través de la resolución de problemas, analizándolo posteriormente bajo los criterios establecidos en las matrices de evaluación y comprendiendo el proceso de aprendizaje a través de la teoría existente.

Como uno de los objetivos es el diseño de los instrumentos de evaluación para la aplicación de la estrategia resolución de problemas, se determinaron los siguientes criterios:

- Planteamiento de situaciones problémicas teniendo en cuenta destrezas $\mathrm{y}$ actitudes de los estudiantes.

- Obtención de información en la cual los estudiantes indagarán a través de diferentes fuentes de información.

- Diseño de estrategias (plan de acción) o estructuración de un plan para la resolución del problema.

- Coherencia, tarea que requiere profundización de los conocimientos y consolidación de los mismos.

- Metodología de intervención en el aula según la propuesta de un plan de acción.

- Análisis de resultados a través de la elaboración de conclusiones.

De otra parte, el instrumento de evaluación para la participación en el foro virtual se realizó con base en la rúbrica Tigre.

Para analizar cómo se desarrolla el aprendizaje a través de la resolución de problemas en un foro virtual, se tendrán en cuenta las características del aprendizaje centrado en el estudiante, pues la participación en los procesos requiere compromiso del mismo.

\section{Población y muestra}

La población está delimitada por los docentes en formación pertenecientes al grupo 500 (Tunja) del séptimo semestre del Programa de Licenciatura en Educación Básica, conformado por 33 estudiantes. 


\section{Sandra Lucía Guerra Gómez y Ariel Adolfo Rodríguez Hernández}

Aprendizaje a través de resolución de problemas en el foro virtual con docentes en formación caso: ciencias naturales, área de educación ambiental, artículo producto de la investigación

La decisión de muestreo en el proceso de investigación se toma para realizar la presentación de los hallazgos (Flick, 2004), es decir se toma una muestra no aleatoria de la información recolectada de las participaciones o intervenciones de los docentes en el foro virtual, antes y después de conocer los criterios de evaluación a través de las matrices establecidas. Seguidamente se procede a la interpretación y explicación del fenómeno. Se realiza el registro y transcripción de las intervenciones producto de la participación de los docentes en formación en el foro virtual.

\section{Presentación de hallazgos}

En síntesis, el instrumento para la presentación de hallazgos fue una ficha descrita en la tabla 2, que se utilizó para la transcripción de las intervenciones y permitió analizar y procesar los datos obtenidos según las necesidades y objetivos definidos por el proyecto.

Tabla 2. Ficha para la transcripción de textos de los estudiantes

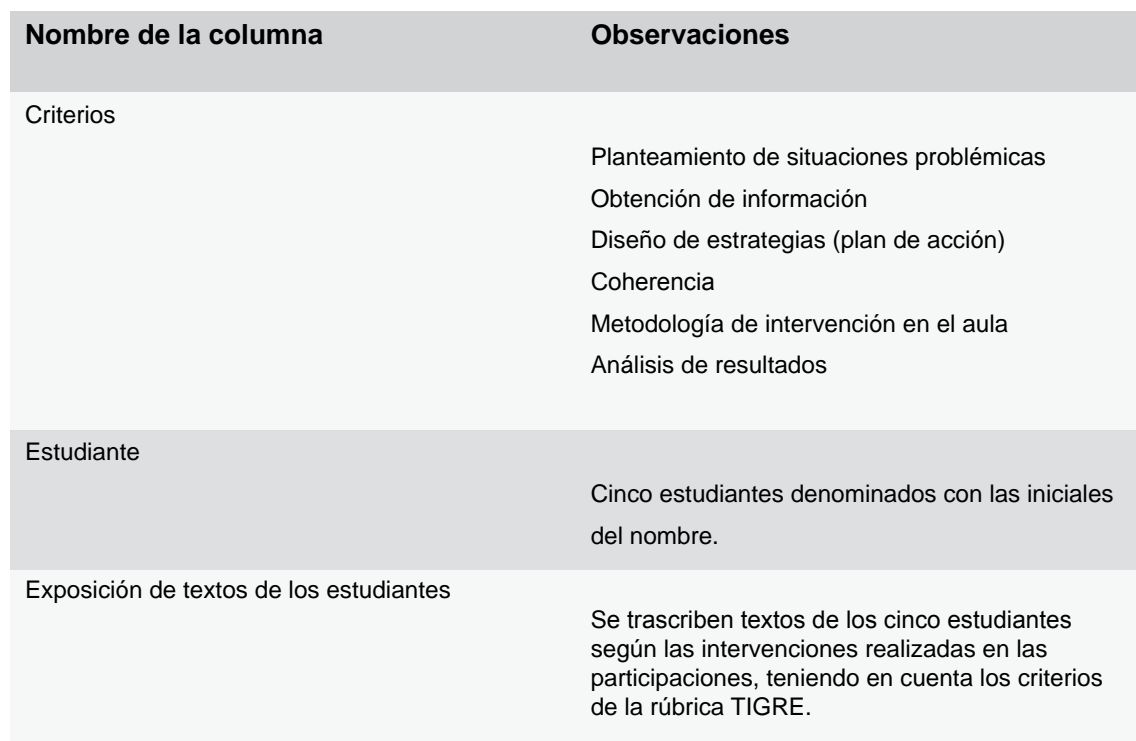

\section{Resultados}

De los 33 estudiantes inscritos en el cursos virtual y que participaron en el foro virtual, 26 de ellos participaron en los tres foros (social de experiencias, de trabajo por proyectos y el foro final). La participación alcanzó el 78.78\%, como revela la tabla 3 . 
Tabla 3. Ingreso de los participantes en el foro virtual

\begin{tabular}{lll} 
Ingreso & Total & Porcentaje \\
\hline Ingresaron a los foros & 26 & $78.8 \%$ \\
No ingresaron a los foros & 7 & $21.2 \%$ \\
Total participantes & 33 & $100 \%$ \\
\hline
\end{tabular}

El promedio de participación en el foro virtual de manera mensual por parte de los 26 participantes se encuentra reflejado en la tabla 4.

Tabla 4. Promedio de participación mensual en el foro

\begin{tabular}{lll} 
Participación & Total & Porcentaje \\
\hline Marzo & 26 & $50 \%$ \\
Abril & 4 & $7.7 \%$ \\
Mayo & 6 & $11.5 \%$ \\
Junio & 16 & $30.8 \%$ \\
Total & 52 & $100 \%$ \\
\hline
\end{tabular}

El promedio de participación en cada foro virtual prueba de manera mensual la participación en cada uno de los foros, notándose en el mes de junio la carencia de participación en el foro social. Esto indicó un aspecto negativo que mostró aislamiento del participante y/o pérdida de interés en el proceso, aspecto a tener en cuenta por parte de la investigadora en el sentido de motivar al estudiante a continuar con él.

Por tanto, la motivación en los participantes se debe incentivar para que sean autores directos de su propio aprendizaje. Hay que invitarlos a una participación dinámica, romper con monólogos o individuos que acaparan la participación, responder y retroalimentar las actividades del grupo, indicando claramente desde un comienzo las expectativas y la metodología de calificación a usar para cada participación individual. Se hacen estos planeamientos siguiendo a Scagnoli (2005), ya que él propone mantener la motivación en los alumnos animándolos para que sean participantes directos de su aprendizaje.

Tabla 5. Promedio de participación en cada foro virtual

\begin{tabular}{|l|l|l|l|l|l|l|}
\hline Conexión & \multicolumn{2}{l|}{$\begin{array}{l}\text { Foro social de } \\
\text { (experiencia) }\end{array}$} & \multicolumn{2}{l|}{$\begin{array}{l}\text { Foro de trabajo por } \\
\text { proyectos }\end{array}$} & \multicolumn{2}{l|}{ Foro final } \\
\hline & Total & Porcentaje & Total & Porcentaje & Total & Porcentaje \\
\hline Marzo & 14 & $77.8 \%$ & 11 & $61.1 \%$ & 1 & $6.25 \%$ \\
\hline
\end{tabular}


Aprendizaje a través de resolución de problemas en el foro virtual con docentes en formación caso: ciencias naturales, área de educación ambiental, artículo producto de la investigación

\begin{tabular}{|l|l|l|l|l|l|l|}
\hline Conexión & \multicolumn{2}{l|}{$\begin{array}{l}\text { Foro social de } \\
\text { (experiencia) }\end{array}$} & \multicolumn{2}{l|}{$\begin{array}{l}\text { Foro de trabajo por } \\
\text { proyectos }\end{array}$} & \multicolumn{2}{l|}{ Foro final } \\
\hline & Total & Porcentaje & Total & Porcentaje & Total & Porcentaje \\
\hline Marzo & 14 & $77.8 \%$ & 11 & $61.1 \%$ & 1 & $6.25 \%$ \\
\hline Abril & 2 & $11.1 \%$ & 1 & $5.6 \%$ & 1 & $6.25 \%$ \\
\hline Mayo & 2 & $11.1 \%$ & 2 & $11.1 \%$ & 2 & $12.5 \%$ \\
\hline Junio & 0 & $0 \%$ & 4 & $22.2 \%$ & 12 & $75 \%$ \\
\hline Total & 18 & $100 \%$ & 18 & $100 \%$ & 16 & $100 \%$ \\
\hline
\end{tabular}

El ingreso total de los estudiantes a cada foro puede determinar que la participación alcanzada sobre el contenido de las respuestas dadas en los foros es una muestra de la dimensión social, participativa y cognitiva. Esto se refleja en el mayor número de intervenciones en el foro social, donde las experiencias de los estudiantes cobraron importancia en el momento de lograr una interacción comunicativa.

En segundo lugar se encuentran las intervenciones que evidencian el trabajo final pues emergen de las interacciones explícitas (donde se responde directamente a un mensaje), implícitas (donde se refieren a otro mensaje sin nombrarlo) e independientes de cada participante (donde se hace referencia al mismo tema sin comentar otro tipo de referencia) y atestiguan el aprendizaje activo. Es aquí donde se debe resaltar la importancia de los otros foros ya que se refleja finalmente el trabajo que de manera conjunta se ha ejecutado para fortalecer el aprendizaje, además de realizar procesos de autorreflexión y aplicación de las matrices de aprendizaje. Finalmente se encuentra la interacción en el foro de trabajo por proyectos, donde los estudiantes intervinieron para obtener información y trabajar en la manera como se resuelven los problemas. Lo anterior se complementa y se visualiza en la tabla 6 .

Tabla 6. Total de participaciones por foro

\begin{tabular}{lll} 
Nombre del foro & Intervenciones & Porcentaje \\
\hline Experiencia & 148 & $49 \%$ \\
Por proyectos & 36 & $11.9 \%$ \\
Final & 118 & $39.1 \%$ \\
Total & 302 & $100 \%$
\end{tabular}

De acuerdo con los datos anteriores, el aprendizaje se da basado en la interactividad porque por sí mismo el foro virtual no asegura situaciones exitosas de aprendizaje, sino el uso correcto de la tecnología para propiciar interacciones que promuevan la construcción del conocimiento (Peralta \& Díaz, 2009). Esto a su vez fortalece el sentido del constructivismo social que, 
de acuerdo con Marqués (2003), amplía las ideas planteadas por un grupo social que construye su aprendizaje creando, en colaboración, una cultura consistente en compartir contenidos y significados.

En consecuencia, las concepciones iniciales sobre la resolución de problemas con las que los estudiantes participaron en el foro experimentaron cambios que se evidenciaron luego de que los estudiantes conocieran los indicadores de evaluación. Además, se trató de permitir a los estudiantes que desarrollaran procesos de resolución más ricos y complejos, favoreciendo aspectos relacionados con la creatividad y la metacognición.

Se encontró que los participantes mejoraron su aprendizaje, así como la calidad de las intervenciones en los foros mencionados. Fue importante este momento ya que se notó que el participante, después de conocer los criterios de evaluación en el foro por proyectos, participó de manera comprometida en el foro final, demostrando que el aprendizaje se da de una manera responsable con la aprehensión de conocimientos. Con ello se favoreció la autonomía en el acceso a la información, procesándola mediante el uso de sus estrategias cognitivas y metacognitivas en un ambiente virtual que sirvió de espacio didáctico para el desarrollo de estos procesos de aprendizaje.

\section{Caracterización de los resultados}

El análisis pretendió mostrar las características encontradas sobre la manera como se desarrolló el aprendizaje en los docentes participantes de esta investigación, entre las que se destacaron las siguientes: el aprendizaje se centró en el estudiante; se estimuló el aprendizaje activo; se permitió que los estudiantes realizaran mejoras continuas; el diseño comprometió al estudiante activamente a "hacer" en lugar de únicamente aprender "sobre" algo.

La gráfica 1 explica las características halladas sobre la manera como se dio el aprendizaje, las cuales, para tener mayor claridad, es necesario conceptualizar por separado.

El aprendizaje se centró en el estudiante, ya que se observó la motivación y el interés en dedicar tiempo y esfuerzo para definir la resolución del problema propuesto. Además, fue notoria la preocupación por la presentación, por la participación y por la construcción de nuevos conocimientos y habilidades en un ambiente virtual asincrónico. Los participantes realizaron indagaciones empleando múltiples fuentes de información tales como Internet, libros y sus propias experiencias, entre otras. Los docentes participaron en la evaluación, en la que alcanzaron una comprensión plena, al aprender a evaluar su propio trabajo y a autorregular sus conocimientos. 


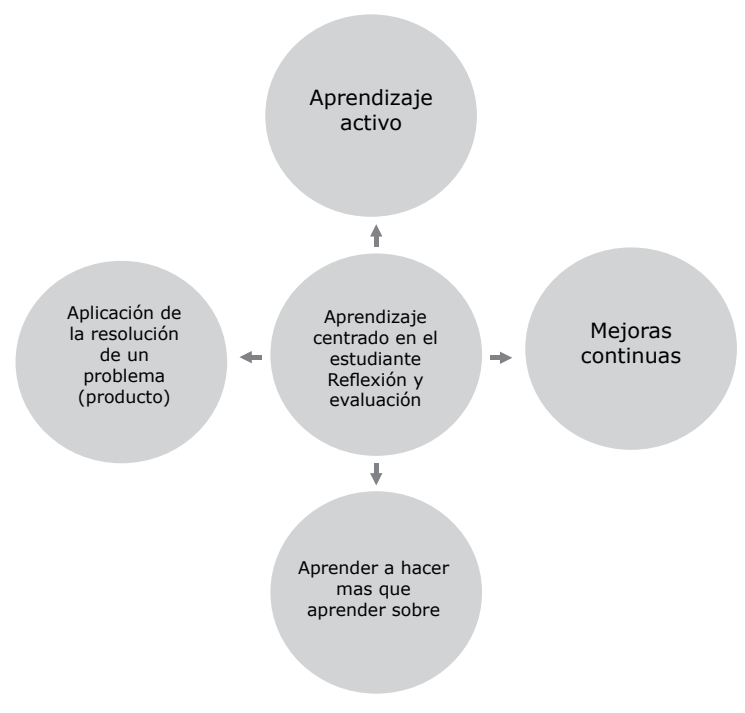

Gráfica 1. Caracterización del aprendizaje en los y las docentes en formación

Al hacer un cuestionamiento del papel que debemos asumir como docentes, es preciso ver lo que Collins (1998), citado por Miranda (2004), plantea para el caso de la educación a distancia: "el centro debe dejar de ser el docente para dar paso al uso de la didáctica". Lo anterior permite inferir que el docente, desde el comienzo, debe ser capaz de determinar las expectativas, necesidades e intereses de los participantes, y para ello las estrategias utilizadas para que se dé la interacción entre ambos serán un aspecto fundamental a tener en cuenta para llevar a feliz término los procesos.

El aprendizaje fue activo debido a que los participantes trabajaron en los diferentes componentes de una tarea y sus esfuerzos de colaboración conjunta se coordinaron mediante la discusión en el foro. Los participantes aprendieron a aprender el uno del otro y a ayudar a que sus compañeros aprendieran. Así mismo evaluaron el trabajo y realizaron retroalimentación constructiva tanto para ellos mismos como para sus compañeros.

Lo anterior permite confirmar lo mencionado por Edith Litwin (2005), quien señala que para adaptarse a los desarrollos tecnológicos el hombre debe tener capacidad para identificar y desplegar actividades cognitivas nuevas, en tanto las tecnologías permanentemente van generando distintas posibilidades; de ahí su condición particular de herramienta, por lo que se reafirma la importancia de su uso. Esto se complementa con la utilización de materiales que contienen toda la información, estructura, secuencia y elementos de retroalimentación para aprender un contenido de modo significativo (Barberà, 2004; Barberà y Rochera, 2008). 
De otra parte, se observó el compromiso de los participantes en "hacer" más que en aprender "sobre algo". Se comprobó la necesidad de comunicarse, compartir y ayudarse en el ambiente de aprendizaje, donde se suministró retroalimentación a través de los criterios de evaluación y los estudiantes aprendieron a depender más de ellos mismos y a aclarar las cosas por sí mismos.

En cuanto a la realización de un producto, éste debía incluir el diseño y presentación que otros pudieran ver o conocer a través de las participaciones. Esto cobró un valor importante ya que ofreció una información complementaria a lo que los demás realizaron. Además, permitió a los estudiantes experimentar, realizar aprendizaje a través de la resolución de un problema, aprender de sus errores y enfrentar y superar retos difíciles e inesperados.

Como los resultados cobran sentido a la luz de "la resolución de problemas" y "los aportes según la rúbrica Tigre", es importante señalar que la descripción se va dando de forma circunstancial, sin separar dentro del análisis las dos categorías. Por el contrario, su presentación permite evidenciar la materialización del aprendizaje alrededor de un proceso. Hay, por tanto, consonancia con la metodología abordada, pues la información recolectada cobra validez cuando, desde su participación, los docentes en formación empiezan a generar aprendizaje.

Teniendo como base el desarrollo de la estrategia resolución de problemas a través del foro virtual, y particularmente su valor pedagógico, existe la posibilidad y la ventaja de discutir alrededor de diferentes contenidos pues en el contexto educativo el solo hecho de discutir es de por sí una estrategia efectiva de aprendizaje, toda vez que es necesario articular y comunicar ideas, así como escuchar y valorar las de los otros, tal como lo señalan Argüelles y Nofal (2007).

En consecuencia, el aprendizaje se desarrolló a partir de la exigencia de los estudiantes de ir más allá de la información dada, con el propósito de crear algo nuevo reconfigurado, expandiendo y aplicando lo que ya saben y construyendo a partir de esos conocimientos. Esta circunstancia a su vez brinda tanto al investigador como a los compañeros la oportunidad de constatar el desarrollo de la comprensión de una temática a lo largo del tiempo y en situaciones nuevas para el aprendizaje.

Una parte importante del proceso de aprendizaje y que debe contribuir significativamente a él es la evaluación. Esta promueve la comprensión del proceso de aprendizaje y tiene que ser algo más que "un examen al final de una unidad". Estas valoraciones deben informar a los estudiantes y a los tutores la comprensión que se han alcanzado y cómo proceder en la enseñanza y el aprendizaje posteriores. Por esta razón fue importante diseñar instrumentos de evaluación del aprendizaje donde se definieron criterios a través de matrices 
evaluativas, que se dieron a conocer a los estudiantes antes de participar en los foros y con base en la resolución de un problema ambiental de estudio.

En relación con la estrategia de resolución de problemas se propuso la resolución del mismo problema ambiental señalado anteriormente, aprovechando los aportes de los estudiantes. Además se elaboró una estrategia de intervención pedagógica en el aula, con los pasos de la resolución de un problema y con todos sus detalles de presentación; esto es, título, descripción y planteamiento del problema, justificación, objetivos, marco teórico, metodología o estrategia de intervención en el aula y conclusiones de la información obtenida, haciéndolo de manera creativa y en respuesta a las expectativas de los participantes.

Se encontró además que los estudiantes planearon situaciones problema teniendo en cuenta ideas, destrezas y actitudes, lo que despertó el interés en el desarrollo de las actividades. Tomaron decisiones para acotar el problema propuesto y orientaron el tratamiento científico del problema incluyendo la invención de conceptos y elaboración de estrategias de intervención en el aula, analizando con coherencia el cuerpo de conocimientos. Plantearon el manejo de nuevos conocimientos en diversas situaciones para profundizar y afianzar los mismos, enfatizándolos en la relación ciencia/tecnología/sociedad, enmarcada en el desarrollo científico. Las actividades de síntesis (presentación del aporte de manera creativa) y la elaboración del resultado final rompieron con el planteamiento de tareas escolares, permitiendo a su vez la concepción de nuevos problemas que pueden ser solucionados en su propio contexto.

A partir de la autogestión del aprendizaje, el estudiante - como bien lo expresa Dewey (1993) — se educó y formó en cuanto tuvo la oportunidad para reflexionar críticamente sobre lo que hizo. Esta reflexión crítica, según Rolheiser et al. (2000), conllevó el desarrollo de la metacognición, del pensamiento crítico, de habilidades en solución de problemas y toma de decisiones y de autoconcepto. Ellos interiorizaron estas operaciones mentales que les facilitaron las herramientas requeridas para la autogestión eficiente de su proceso de aprendizaje.

De otra parte, se ha querido expresar en este trabajo que los estudiantes, además de participar en los foros, debieron hacerlo aportando sus ideas construidas a partir de los criterios establecidos para lo cual recibieron indicaciones sobre la manera de hacer sus intervenciones.

Éstas se dirigieron hacia la solución de interrogantes compartidos por el grupo y su retroalimentación, creando la posibilidad de poner en tensión las interpretaciones de la realidad educativa o del contexto del estudiante, a la luz de la teoría en la que el estudiante se basó y la sistematización de las intervenciones. Un denominador común fue la construcción de una estrategia 
de intervención en el aula que permitió puntualizar los aportes teóricos del material bibliográfico.

\section{Conclusiones}

Surgen de los resultados obtenidos a partir del análisis del aprendizaje generado con la aplicación de la estrategia "resolución de problemas" en el foro virtual. Con ella se logró observar la actitud del estudiante frente a una realidad ambiental, como el uso indiscriminado de los plaguicidas. Se forjó un aprendizaje consciente que significó aprender a aprender, aprender significativamente, aprender activamente y aprender descubriendo, al utilizar una estrategia pedagógica que permitió una participación activa.

Respecto al aprendizaje, los participantes lograron determinar un producto a elaborar, definir un procedimiento implícito, reconocer los recursos con los que se cuenta y el manejo de un monitoreo. Así se produjeron cambios en la manera como se avanzó en la producción de conocimiento y su autorregulación.

Se evidenció el desarrollo de habilidades en el uso de un ambiente virtual, al mismo tiempo que hubo una aproximación a la meta propuesta para alcanzar la autorregulación del proceso de aprendizaje, ya que el estudiante participó de manera proactiva en la construcción de los conocimientos haciendo referencia a las ideas, sentimientos y actos generados por el mismo y que se orientan de manera sistemática a la consecución de metas.

En las intervenciones de los estudiantes se mostró que internalizaron el proceso, es decir, apropiaron paulatinamente las instrucciones, ideas, estructuras, conocimiento y prácticas que estaban fuera de su pensamiento, y de esta manera alcanzaron el dominio autónomo de lo aprendido. Además se evidenció la interiorización de su aprendizaje, ya que el estudiante construyó conocimiento basado en las ideas de otros, para finalmente trasferir un proceso. Se implementó la estrategia resolución de problemas para lograr el aprendizaje, para lo cual se les indicó a los estudiantes cómo enfrentarse en el aula a problemas de su entorno. Se les motivó sobre la forma de pensar y actuar ante situaciones que se pueden resolver sin incertidumbre. Se demostró que no se enseña a resolver problemas sino que se muestran los diferentes caminos que se pueden tomar para dar soluciones a problemas reales, según los conocimientos que se tienen de las temáticas identificadas.

En lo concerniente a la práctica docente, no puede reducirse a un asunto instrumental y de manejo de técnicas; se trata de conocer a fondo la complejidad de los procesos de construcción y reconstrucción del conocimiento inherente a la enseñanza. La mediación pedagógica busca abrir el camino a nuevas relaciones 
del estudiante con los materiales y recursos ofrecidos para su aprendizaje, relaciones que son también con el propio contexto, con sus compañeros de aprendizaje, incluido el docente, consigo mismo y con su futuro.

Respecto al desarrollo del foro virtual, el proceso de aprendizaje se cumplió, evidenciándose la adquisición y construcción de conocimiento, por cuanto los estudiantes recibieron información valiosa de sus compañeros de foro. Además demostraron la interacción, reflejando con sus mensajes la intención y el contenido de los mismos, aprovechando o utilizando ideas de otros o de fuentes de información que hicieron más profundas sus intervenciones. Hay preocupación por escribir y redactar de manera adecuada los textos que a su vez generaron discusión, teniendo en cuenta las implicaciones de motivación, pertinencia e integración a una comunidad virtual de aprendizaje que lleva a crear responsabilidades para que otros aprendan.

Se desarrolló el aprendizaje a través de las relaciones entre todos los participantes, la resolución de dudas, la participación en la discusión y la motivación a los participantes, de manera que se aseguró el fortalecimiento de los procesos de aprendizaje.

La utilización del aula virtual y el foro virtual facilitó y fortaleció el aprendizaje autónomo y el aprendizaje colaborativo, ya que se posibilitó la interacción entre los participantes en la búsqueda del conocimiento hacia metas comunes, logrando desarrollar nuevos conocimientos. En desarrollo de la estrategia se aclararon dudas, se compartieron experiencias y se llegó a conclusiones sobre un tema en particular. Esto favoreció el aprendizaje de tal manera que trasciende la costumbre de adquirir información, procesarla e incorporar nuevas destrezas y conocimientos, dirigiéndose al logro de objetivos comunes.

Finalmente, la evaluación de los procesos de aprendizaje fue fundamental para que los participantes superaran sus dificultades, posibilitando la aclaración de dudas y la retroalimentación inmediata en sus avances y tropiezos. Así el docente deja de ser el que transmite el conocimiento para convertirse en un evaluador permanente del aprendizaje de cada uno de sus estudiantes, a los cuales valora sus ejecuciones para consolidar o reorientar los logros.

\section{Referencias bibliográficas}

Argüelles, D. C., Nofal, N. (2007). Estrategias para promover procesos de aprendizaje autónomo. Colombia: Alfaomega.

Baracat, María Paula; Ginocchio, María Virginia. (2006). Debates, reflexiones e interrogantes: el foro como propuesta de articulación entre espacios curriculares 
en la educación a distancia en entornos asincrónicos. Cultural Inglesa de Buenos Aires, Argentina.

Barberá, E. (2004). La educación en red: Actividades virtuales de enseñanza y aprendizaje. Barcelona: Paidós.

Barberá, E, Y M. J Rochera. (2008). Los entornos virtuales de aprendizaje basados en el diseño de materiales autosuficientes y el aprendizaje autodirigido. En Psicología de la educación virtual, editado por C. Coll y C. Monereo. España: Morata.

Coll, C. \& Solé, I. (2001). Enseñar y aprender en el contexto del aula. En: C. Coll, J. Palacios y A. Marchesi (Comps.). Desarrollo Psicológico y Educación. Vol. 2. Psicología de la Educación Escolar. Madrid: Alianza.

Collison, G., Elbaum, B., Haavind, S., And Tinker, R. (2000). Aprendizajes en Ambientes Virtuales. Concord, MA: The Concord Consortium. (mimeografiado).

Dewey, J. (1993). Cómo pensamos. Boston. D.C. Heath Company.

Flick, U. (2004). Introducción a la investigación cualitativa. Ediciones Morata, Madrid.

Galvis, A. (2008). Criterios y rúbrica TIGRE para autocontrolar calidad de aportes en discusiones. Disponible en http://aportetigre.blogspot.com.

Gil, D., Dumasumas-Carré, A., Caillot, M., Martínez Torregrosa, J. \& Ramírez, L. (1989). La resolución de problemas de lápiz y papel como actividad de investigación. Investigación en la escuela, 6.

Litwin, Edith (Comp.). (2005). La educación a distancia. Temas para el debate en una nueva agenda educativa, Buenos Aires: Amorrortu.

Marqués, P. (2003). Usos educativos de Internet. ¿Hacia un nuevo paradigma de la enseñanza? Departamento de Pedagogía Aplicada. Facultad de Educación. UAB. En http://dewey.uab.es/pmarques/usosred2.htm.

Mercer, N. (2001). Palabras y mentes: Cómo usamos el lenguaje para pensar juntos. Barcelona: Paidós.

Miranda Díaz, Germán Alejandro. (2004). De los ambientes virtuales de aprendizaje a las comunidades de aprendizaje en línea. Revista Digital Universitaria [en línea]. 10 de noviembre de 2004, Vol. 5, No. 10. Disponible en http://www.revista.unam.mx/vol.5/ num10/art62/int62.htm ISSN: 1607-6079.

Peralta A. \& Díaz Barriga F. (2009). Diseño instruccional de ambientes virtuales de aprendizaje desde una perspectiva constructivista. 


\section{Sandra Lucía Guerra Gómez y Ariel Adolfo Rodríguez Hernández}

Aprendizaje a través de resolución de problemas en el foro virtual con docentes en formación caso: ciencias naturales, área de educación ambiental, artículo producto de la investigación

Universidad Nacional Autónoma de México. Consultado el 17 demarzo de 2010 en: http:// www.gabinetecomunicacionyeducacion.com/files/adjuntos/Diseñolnstruccional de ambientes virtuales de aprendizaje desde una perspectiva constructivista.pdf.

Rolheiser, C; Bower, B. Y Stevahn, L. (2000). The portfolio organizer: succeeding with portfolios in your classroom. Association for supervision and curriculum development (ASCD). Canadá. [Libro en línea]. Disponible: http://www.ascd.org/readingroom/ books/ rolheiseroobook.html.

Scagnoli, N. (2005). Estrategias para motivar el aprendizaje colaborativo en cursos a distancia. College of Education, University of Illinois at Urbana-Champaign, USA. 